\title{
Objective probability-like things with and without objective indeterminism
}

\author{
László E. Szabó \\ Theoretical Physics Research Group of HAS \\ Department of Logic, Institute of Philosophy \\ Eötvös University, Budapest \\ http://philosophy.elte.hu/leszabo
}

(Preprint)

\begin{abstract}
I shall argue that there is no such property of an event as its "probability." This is why standard interpretations cannot give a sound definition in empirical terms of what "probability" is, and this is why empirical sciences like physics can manage without such a definition. "Probability" is a collective term, the meaning of which varies from context to context: it means different-dimensionless [0,1]-valuedphysical quantities characterising the different particular situations. In other words, probability is a reducible concept, supervening on physical quantities characterising the state of affairs corresponding to the event in question.

On the other hand, however, these "probability-like" physical quantities correspond to objective features of the physical world, and are objectively related to measurable quantities like relative frequencies of physical events based on finite samples-no matter whether the world is objectively deterministic or indeterministic.
\end{abstract}

Key words: probability, interpretation of probability, branching space-time, quantum probability

\section{Introduction}

One of the central issues in the recent branching space-time literature is how to integrate the concept of single-case probability into the modalcausal description of the world. (Weiner and Belnap 2006; Müller 2005.) Investigating this problem within the formally rigorous framework of 
branching space-time theory immediately raises the old fundamental question about interpretation of probability. Let me demonstrate the essence of the problem.

Consider the following typical probabilistic assertions: In quantum mechanics,

$$
p(a)=\operatorname{tr}\left(\hat{P}_{a} \hat{W}\right)
$$

asserts that the probability that the value of a physical quantity falls into a Borel set $a$ is equal to $\operatorname{tr}\left(\hat{P}_{a} \hat{W}\right)$ where $\hat{W}$ is the state operator of the system and $\hat{P}_{a}$ denotes the corresponding projector. Formula

$$
p\left(\left\{N_{i}\right\}_{i=1,2, \ldots}\right)=\frac{\left(\sum_{i} N_{i}\right) !}{\prod_{i} N_{i} !}
$$

in statistical mechanics asserts that the probability that the microdistribution is equal to $\left\{N_{i}\right\}_{i=1,2, \ldots .}$ is equal to $\frac{\left(\sum_{i} N_{i}\right) !}{\prod_{i} N_{i} !}$. The meteorologist claims that the probability that it will be raining tomorrow is

$$
p(\text { raining })=0.8
$$

A simple probabilistic description of a coin-flip claims that the probability of getting Heads is

$$
p(\mathrm{H})=\frac{1}{2}
$$

Let us compare these assertions with other scientific assertions. The electric field strength of a point charge $q$ :

$$
\mathbf{E}(\mathbf{r})=q \frac{\mathbf{r}-\mathbf{r}_{q}}{\left|\mathbf{r}-\mathbf{r}_{q}\right|^{3}}
$$

The time tag of an event $A$ :

$$
t(A)=43 \mathrm{~s}
$$

In case of (5) and (6) it is clear what the formulas assert. For example, on the left hand side of (6) we have a known, previously empirically defined physical quantity, and (6) asserts that the value of this quantity is equal to 43s. Similarly, in (5), when we assert that the static electric field strength of a point charge is $q \frac{\mathbf{r}-\mathbf{r}_{q}}{\left|\mathbf{r}-\mathbf{r}_{q}\right|^{3}}$, we have a previously defined physical quantity, electric field strength, and (5) expresses a contingent fact about this quantity.

It is far from obvious, however, what formulas (1)-(4) actually assert. What quantities are on the left hand sides of (1)-(4)? What is "probability"? In my views, "interpretation of probability" means-or ought to meanthe answering this question. For the aim of interpretation of probability is not, as many believe, to assign meaning to the mathematical terms "probability" or "probability measure" of, say, Kolmogorov's probability theory. 
For, when we define the notion of electric field strength in empirical terms, our aim is to introduce an objective characteristic of electromagnetic field, but not to assign meaning to the mathematical term "vector field". So the right epistemological order would be something like this:

1. We have to define-in empirical terms-what "probability of an event" means on the left hand side of (1)-(4) and in other similar scientific assertions.

2. From the knowledge of (1)-(4) and other similar facts, acquired by $a$ posteriori means, we ascertain the basic laws satisfied by the quantity we previously defined and called "probability".

3. Finally, we may conclude-again, on the basis of our observationsthat probability can be conveniently described by the mathematical concept called "probability" in Kolmogorov's "probability theory".

Although all standard interpretations-the classical, the frequency, the propensity, and the subjective interpretations-can grasp something from our intuition about probability, there is a consensus that none of them can provide an ultimate definition of what probability is. (Earman and Salmon 1992; Hájek 2003.) According to this consensual conclusion we have the following

\section{Stipulations}

(A) Probability is not the ratio of cases favourable to the event in question over the total number of (equally possible) possibilities.

(B) Probability is not relative frequency on a finite sample.

(C) Probability is not limiting relative frequency.

(D) Probability is not propensity.

(E) Probability is not degree of belief.

Then what is probability? And how is it possible then that physics and other empirical sciences apply a formal (mathematical) theory of probability, without noticing a problem arising from this unanswered fundamental question? In this paper I shall make an attempt to develop a new interpretation of probability, which may perhaps throw light on this matter. 


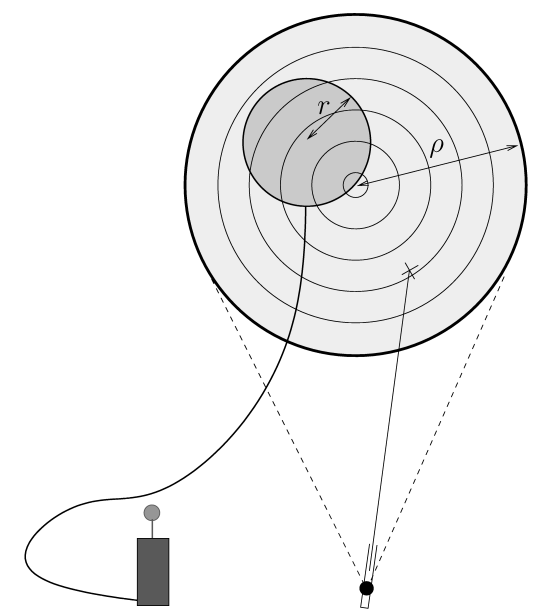

Figure 1: A gun is hinged in such a way that it can fire uniformly at a round target area, radius $\rho$, with an inflated balloon, radius $r$, attached to the front of the target

\section{'No-probability' Interpretation of Probability}

The key idea of my proposal, which I call 'no-probability' interpretation of probability, is that there is no such property of an event as its "probability". If there is any reason to use this word, "probability" is merely a collective term: its meaning varies from context to context. Moreover, these contextdependent meanings reduce the concept of "probability" to ordinary physical quantities. This is why standard interpretations fail to give a sound definition of probability, and this is why empirical sciences like physics can manage without such a definition.

From philosophical point of view, my argument will be based on the following two general principles: One is a kind of verificationist theory of meaning, the second is a (non-mathematical) indispensability argument.

I shall rely on the verificationist theory of meaning in the following very weak sense: In physics, and in other empirical sciences, the meaning of a term standing for a quantity which is supposed to characterise an objective feature of (physical) reality is determined by the empirical operations with which the value of the quantity in question can be ascertained.

The indispensability argument claims that we ought to have ontological commitment to all and only the entities that are indispensable to our best scientific theories. Mutatis mutandis, we ought to have ontological commitment to all and only the features of reality that are indispensable to our best scientific theories.

Consider the following example. A gun is hinged in such a way that it can fire uniformly at a round target area, radius $\rho$, with an inflated balloon, 
radius $r$, attached to the front of the target. (Fig. 1). What is the probability that the balloon will be burst (event $A$ )?

The physicist's standard answer to this questions is the following:

$$
p(A)=\frac{\pi r^{2}}{\pi \rho^{2}}
$$

We will not look at how the physicist arrives at this result. What is important is that this equation does not, cannot, express a contingent fact of nature. The right hand side of (7) is meaningful. It is an expression consisting of known physical quantities. On the left hand side, however, $p(A)$ is not a known quantity which could be contingently equal to $\frac{\pi r^{2}}{\pi \rho^{2}}$. There is no way to test empirically whether equality (7) is correct or not. Many believe that this is possible by measuring the relative frequency of $A$ and testing that $\frac{N(A)}{N} \approx \frac{\pi r^{2}}{\pi \rho^{2}}$. Beyond the problem that relative frequency $\frac{N(A)}{N}$ has, in general, nothing to do with $\frac{\pi r^{2}}{\pi \rho^{2}}$ (see below), the main objection to this argument is that probability is not relative frequency (Stipulation (B)-(C)). So the only possible interpretation of equation (7) is that it is a definition of $p(A)$ :

$$
p(A) \stackrel{\text { def }}{=} \frac{\pi r^{2}}{\pi \rho^{2}}
$$

Note that physical quantity $\mu(\ldots)=\frac{\text { area of } \ldots}{\pi \rho^{2}}$ happens to be a "probability like" quantity. It is a dimensionless normalised measure, satisfying the Kolmogorov axioms.

In case of a completely different scenario, "probability" is defined as a dimensionless normalised measure composed by completely different physical quantities. For example,

$$
\begin{aligned}
p(a) & \stackrel{\text { def }}{=} \operatorname{tr}\left(\hat{P}_{a} \hat{W}\right) \\
p\left(\left\{N_{i}\right\}_{i=1,2, \ldots}\right) & \stackrel{\text { def }}{=} \frac{\left(\sum_{i} N_{i}\right) !}{\prod_{i} N_{i} !}
\end{aligned}
$$

Therefore, "probability", at best, can be used only as a collective term the meaning of which varies from context to context. To sum up:

Thesis 1 There is no such property of an event as its "probability." What we call probability is always a physical quantity characterising the state of affairs corresponding to the event in question. "Probability" can be used only as a collective term: it means different dimensionless [0,1]-valued physical quantities, or more precisely, different dimensionless normalised measures composed by different physical quantities in the various specific situations. 


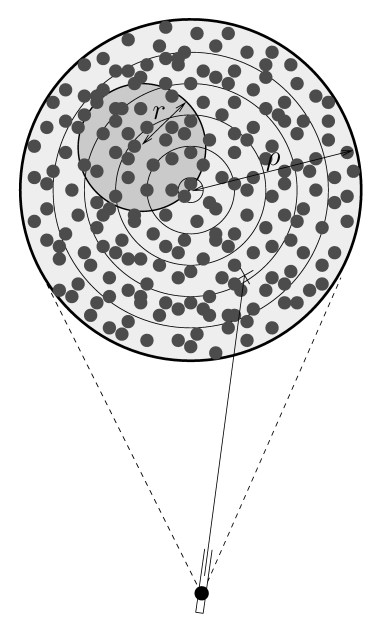

Figure 2: If the size of the balloon is constant and the uniform distribution of the shots on the target is provided then the relative frequency of event $A$ is approximately equal to $\frac{\pi r^{2}}{\pi \rho^{2}}$

For scientific practice, the most important question is how probability is related to relative frequency. In our balloon example, we used the term "probability" for the quantity $\frac{\pi r^{2}}{\pi \rho^{2}}$. The value of $\frac{\pi r^{2}}{\pi \rho^{2}}$ is a definite number in each individual experiment, so it is a meaningful notion for an individual event. Imagine that we change the size of the balloon during the sequential repetitions of the experiment, such that the sequence of relative frequencies cannot converge to a limiting value. In this case, $\frac{\pi r^{2}}{\pi \rho^{2}}$ has nothing to do with the relative frequency of event $A$. But, consider the following particular case. Let the value of $\frac{\pi r^{2}}{\pi \rho^{2}}$ be constant and let the uniform distribution of shots at the target be ensured (Fig. 2) by setting up the position of the gun with a computer applying a suitable ergodic map. In this particular case, the relative frequency of event $A$ is approximately equal to "probability" $\frac{\pi r^{2}}{\pi \rho^{2}}$. And this fact has nothing to do with probability-theoretic considerations. It is a simple result of elementary kinematics. Generalising this observation, we formulate our next thesis:

Thesis 2 The physical quantity identified with "probability" is not the limiting value of relative frequency, and not even necessarily related to the notion of frequency. In some cases, the conditions of the sequential repetitions of a particular situation are such, however, that the physical quantity called "probability" in the given particular context is approximately equal to the relative frequency of the event in question.

The physical quantity $\frac{\pi r^{2}}{\pi \rho^{2}}$ is meaningful and has a certain value in every 
single run of the experiment. Its existence and value are independent of whether the laws of nature governing the gun firing and the path of the bullets are deterministic or not.

Moreover, the relationship between $\frac{\pi r^{2}}{\pi \rho^{2}}$ and the relative frequency of $A$ (if there is such a relationship at all) is not influenced by the deterministic or indeterministic character of the physical processes in question. The relative frequency will be (approximately) equal to $\frac{\pi r^{2}}{\pi \rho^{2}}$ independently of whether the uniform distribution of the shots is ensured by means of a deterministic ergodic process, or by means of an objectively random firing following a uniform distribution.

In talking about objectively random firing following a uniform distribution, it is necessary to be careful of a possible misunderstanding. One must not think of a kind of "objective chance" of the gun firing in any particular direction being uniform. The problem is not with the "objectivity" of this "chance"-due to the objectivity of the randomness-but with the "chance" (probability) itself. Because there is no "chance" neither objective nor epistemic; according to Thesis 1, the "probability" distribution of the gun firing in the different directions means a dimensionless normalised measure composed by ordinary physical quantities characterising the physical process selecting the different directions-no matter if this process is deterministic or not. Independently of the details of this physical process, the phrase "the distribution of the directions is uniform" simply means that, say, the density of the dots (number of dots $/ \mathrm{cm}^{2}$ ) on the target is uniform. This is an ordinary physical statement about meaningful measurable physical quantities, of the same kind as "this rigid rod is homogeneous", and the likes. And, if the distribution of the directions is uniform then

$$
(p(A) \stackrel{\text { def }}{=}) \frac{\pi r^{2}}{\pi \rho^{2}} \approx \frac{N(A)}{N}
$$

no matter if the process in question is indeterministic or deterministic.

Similarly, neither the value of $\frac{\pi r^{2}}{\pi \rho^{2}}$ nor the relationship (11) can be influenced by anything related to our knowledge about the details of the process. For example, if the uniform distribution of shots condition is satisfied, (11) holds independently of whether we know the direction of the subsequent shot, or not.

Finally, we have to emphasise that it is the real physical process that actually determines whether the distribution of the shots is uniform or not. We must not suppose that the distribution is uniform, a priori, merely because we have no information about how the directions of the consecutive shots are determined and, on this basis, we have no reason to prefer one direction to the other.

So, our last three Theses are the following: 
Thesis 3 The value of the physical quantity identified with "probability" is not influenced by the fact whether the process in question is indeterministic or not. (And, of course, there is no reason to suppose that this value can be only 0 or 1 , merely because the process is deterministic.)

Thesis 4 The value of the physical quantity identified with "probability" is not influenced by the extent of our knowledge about the details of the process.

Thesis 5 Neither the value of the physical quantity identified with "probability," nor the existence of the conditions under which this value and the relative frequency of the corresponding event are approximately equal can be knowable a priori.

Although standard interpretations do not provide a tenable definition of probability, they grasp many important aspects of our intuition of probability. It is remarkable that a physical quantity like $\frac{\pi r^{2}}{\pi \rho^{2}}$ reflects many of these intuitive features:

1. Like classical probability, in some sense, it reflects the ratio of favourable cases to the number of equally possible cases.

2. Like propensity, a) it is meaningful and has a certain value in each individual experiment, b) in some sense it expresses the "measure of the tendency" of the whole system to behave in a certain way, 3) in general, it has nothing to do with relative frequencies.

3. Under suitable circumstances, however, it is approximately equal to the relative frequency measured during the sequential repetitions of the experiment. There are no general conditions ensuring such a relationship; it depends on the particular physical conditions in the given particular context. In our example, we may know-as a fact of kinematics - that equation (11) holds. In this case, the truths about the normalised measure $\mu(\ldots)=\frac{\text { area of } \ldots}{\pi \rho^{2}}$ are in correspondence with the truths about the corresponding relative frequencies $\frac{N(\ldots)}{N}$. This fact can explain and justify the standard rules of statistical practice; more exactly, can explain why these rules are applicable in the given case. For, consider the following claims:

There are such things as the probabilities of events. These probabilities are normalised measures satisfying the Kolmogorov axioms. The whole system of conditions are such that the values of these measures are equal to the corresponding relative frequencies. 
There are no such things as the probabilities of events. "Probabilities" are normalised measures consisting of ordinary physical quantities. These measures satisfy the Kolmogorov axioms. The whole system of conditions are such that the values of these measures are equal to the corresponding relative frequencies.

If the traditional thesis $(\mathrm{T})$ implies and explains the standard rules of statistical practice, then the 'no-probability' thesis $(\mathrm{N})$ also implies and explains the same rules of statistical practice. In our example, one can imagine a situation when we cannot measure the size of the balloon. Given, however, that condition (11) holds, we can ascertain $(p(A) \stackrel{\text { def }}{=}) \frac{\pi r^{2}}{\pi \rho^{2}}$ (that is, the size of the balloon) by measuring the relative frequency $\frac{N(A)}{N}$. Moreover, the statistician may apply, for example, the method of "random sampling", given that the sampling, as a real physical process, is such that the uniform distribution of the samples in the large ensemble is ensured. What is new in the 'no-probability' approach is that we do not justify these methods by saying that "the probability of every direction is the same" or "each element of the ensemble is selected with equal probability", etc. Because we deny that there are such things as probabilities. Instead, we are committed at real physical things like $\frac{\pi r^{2}}{\pi \rho^{2}}, \frac{N(A)}{N}$ (on finite ensembles), kinematical conditions ensuring the uniform distribution of shots, physical conditions providing the uniform distribution of the selected samples in the larger ensemble, etc. And the facts about these real physical things provide enough reason to apply the statistical methods, whenever these methods are applicable.

So far so good. It seems we must, in every context, manifest a physical quantity that corresponds to "probability" in the given context. In this way we can clarify how we should understand expressions like (7), (1), and (2). But how should we understand expressions like (3) and (4)?

Let us continue our above example. Assume we know that $r=\frac{1}{2} \rho$, therefore

$$
p(A) \stackrel{\text { def }}{=} \frac{\pi r^{2}}{\pi \rho^{2}}=\frac{1}{4}
$$

In brief,

$$
p(A)=\frac{1}{4}
$$

That is, (13) is just an incomplete formulation of (12). Statement (13) in itself is completely meaningless, for " $p(A)$ " on the left hand side has no meaning.

Consider statement (4). In order to make sense of it, we must assume that there exists a physical quantity $X$ corresponding to "probability" in the given context, such that

$$
p(\mathrm{H}) \stackrel{\text { def }}{=} \mathrm{X}=\frac{1}{2}
$$


Although the system in question, the coin together with its environment, is a very complex physical system, we may have a good intuition about the system's phase space and the phase space regions corresponding to events Heads and Tails, etc. So we can imagine what kind of physical quantity $X$ might be. On this intuitive level, without knowing any details of the system's dynamics, we have enough reason to apply some symmetry principles, and conclude (with the hypothesis) that $X=\frac{1}{2}$.

Although, the physical quantity $X$, in general, has nothing to do with relative frequency of getting Heads, the conditions of the sequential repetitions of the coin-flip can be such that $X \approx \frac{N(H)}{N}$. And of course, this provides a possibility to test our hypothesis that $X=\frac{1}{2}$. This is, however, not important. Again, what is important is that (4) is meaningless in itself; it must be understood in the form of (14), where $X$ is an ordinary physical quantity.

The meaning of (3) is much less clear. If we take it as a serious probabilistic assertion, then we have to assume that the meteorologist has a (physical) theory on which assertion (3) is based. That is, again,

$$
p(\text { raining }) \stackrel{d e f}{=} X=0.8
$$

for some physical quantity $X$. In practice, however, the meteorologist does not necessarily have such a theory with such an $X$, but (s)he simply asserts the statistical fact that the relative frequency of raining in similar situations, in the past, was 0.8. That is to say, this is not an assertion about "probability" of raining - taking into account Stipulation (B).

\section{A problem}

Let us consider again the quantum mechanical expression (9). First sight it is a correct definition of "probability" on the left hand side, just like in cases of (8) and (10). But, on the right hand side, $" \operatorname{tr}\left(\hat{P}_{a} \hat{W}\right)$ " itself is not a well defined physical quantity having independent empirical meaning. For we can ascertain the state operator $\hat{W}$ only by measuring many different "probabilities" like $p(a)$. But, what is "probability" here? One might think that the value of "probability" can be ascertained by measuring relative frequency, even if we do not know what "probability" exactly is. This is the case, indeed, if we may assume that "probability" is something approximately equal to relative frequency. In quantum mechanics, however, we have no justification for such an assumption whatsoever!

There are two possible reactions to this situation: 1) We can take the position that "probability" is an inappropriate concept for quantum mechanics, and the statistical rules of quantum mechanics are nothing but connections between relative frequencies (on finite samples) relative to 
different measurement setups. 2) We can try to figure out what kind of physical quantity is lurking behind " $\operatorname{tr}\left(\hat{P}_{a} \hat{W}\right)$ ", that is, what kind of non-probabilistic meaning can be assigned to wave function-just like in Bohmian mechanics or in quantum field theory.

The research was partly supported by the OTKA Foundation, No. T043642.

\section{References}

Earman, J. and Salmon, W. (1992). The Confirmation of Scientific Hypotheses, In M. H. Salmon, et al., Introduction to Philosophy of Science, Prentice Hall, Englewood Cliffs, New Jersey.

Hájek, A. (2003). Interpretations of Probability, The Stanford Encyclopedia of Philosophy (Summer 2003 Edition), Edward N. Zalta (ed.), http://plato.stanford.edu/archives/sum2003/entries/probabilityinterpret.

Müller, T. (2005). Probability theory and causation. A branching space-times analysis, Br. J. Philos. Sci., 56, 487-520.

Weiner, M. and Belnap, N. (2006). How causal probabilities might fit into our objectively indeterministic world, Synthese, 149, 1-36. 